\title{
Article \\ Design and Performance Analysis of the Hydropneumatic Suspension System for a Novel Road-Rail Vehicle
}

\author{
Bonan Qin ${ }^{1}\left(\mathbb{D}\right.$, Riya Zeng ${ }^{1}$, Xiaoman $\mathrm{Li}^{2}$ and Jue Yang ${ }^{1, *(\mathbb{C})}$ \\ 1 Department of Mechanical Engineering, University of Science and Technology Beijing, Beijing 100083, China; \\ bonanqin@hotmail.com (B.Q.); zengriya@gmail.com (R.Z.) \\ 2 CRRC Academy Co., Ltd., Beijing 100070, China; xiaoman907@126.com \\ * Correspondence: yangjue@ustb.edu.cn
}

check for updates

Citation: Qin, B.; Zeng, R.; Li, X.; Yang, J. Design and Performance Analysis of the Hydropneumatic Suspension System for a Novel Road-Rail Vehicle. Appl. Sci. 2021, 11, 2221. https://doi.org/10.3390/ app11052221

Academic Editor: Nicola Bosso

Received: 23 December 2020

Accepted: 26 February 2021

Published: 3 March 2021

Publisher's Note: MDPI stays neutral with regard to jurisdictional claims in published maps and institutional affiliations.

Copyright: (c) 2021 by the authors. Licensee MDPI, Basel, Switzerland. This article is an open access article distributed under the terms and conditions of the Creative Commons Attribution (CC BY) license (https:// creativecommons.org/licenses/by/ $4.0 /)$.

\begin{abstract}
Road-rail vehicles built on traditional vehicle chassis can only switch operation modes at particular areas such as level crossings, thus limiting the working scope and efficiency of routine railway inspection and maintenance. This paper proposes a novel tracked chassis for the roadrail vehicle with a multi-cylinder hydropneumatic suspension system, which can better adapt to rough terrains and enhance the vehicle ride performance. Based on this hydropneumatic suspension design, the single-cylinder mathematical model is derived and validated by experimental data. An in-plane multi-body dynamics (MBD) model and road model are established, combined with the hydropneumatic suspension model, including the LuGre friction force. Virtual tests are conducted to investigate the effects of different initial gas volumes, varied diameters and damping pipe lengths on the ride performance. The results indicate that improper damping pipe diameter and charge gas volume will deteriorate the ride performance, which provides a useful reference for the optimization design and control of the hydropneumatic system.
\end{abstract}

Keywords: suspension; vehicle dynamics; ride performance; tracked chassis

\section{Introduction}

Differing from general vehicles operating on prepared roads, special vehicles equipped with tracked chassis are designed for negotiating harsh terrains. The irregular loads and impacts transmitted from such uneven terrains lead to maneuverability loss and ride performance degradation. Hence, the hydropneumatic suspension is widely used in offroad tracked vehicles, not only because of its good non-linear elastic stiffness and superior damping performance [1] but also for the high-power density with lightweight. With such good properties, the tracked vehicles can better handle the conflict between the ride comfort and handling stability than ordinary trailing arm suspension $[2,3]$.

The characteristics of hydropneumatic suspension have already attracted wide research attention in wheeled vehicles. Yin et al. [4] studied the hydropneumatic suspension implemented on the front axle of an articulated vehicle by sensitivity analysis and optimization for the design parameters. The results suggested that the hydropneumatic suspension can help preserve directional stability and reduce driving vibration by nearly $30 \%$. Nieto et al. [5] presented an analytical model of air spring suspensions and obtained its first-order Taylor series linearized model, both of which are validated with experimental measurements. Gobbi et al. [6] discussed the hydropneumatic suspension characteristics of a $6 \times 6$ amphibious vehicle. A mathematical model of the whole vehicle, including thermal transfer of the actuator and the hydraulic circuit, has been realized and experimentally tuned. Martini et al. [7] presented a highly non-linear hydropneumatic suspension with strong regressive spring stiffness for motorcycles. By connecting the hydropneumatic suspension and the spring in series, the grip can be increased while ensuring that the comfort level is basically unaltered. These publications based on wheeled vehicles provide 
effective theoretical methods and models for the research of hydropneumatic suspension for tracked vehicles.

Current studies on the hydropneumatic suspension of tracked vehicles mainly focus on heavily armored vehicles. Cho and Lee [8] investigated the non-linear elastics and the damping characteristics of an in-arm hydropneumatic suspension unit (ISU). The results showed that the desirable vertical displacement could be achieved by adjusting both the orifice diameter and pre-displacement of disc springs. Solomon and Padmanabhan [9] validated the mathematical model of a hydro-gas suspension with experimental data. This model was incorporated in an in-plane model and the influence of suspension parameters were studied for different sinusoidal inputs. Although different hydropneumatic suspension designs and configurations have been proposed, their ride performance analysis methods still rely on the traditional analysis methods served for wheeled vehicles.

The systematic dynamics research for the ride performance analysis on various road surfaces of tracked vehicles started in the 1990s. Wong [10,11] and McCullough and Huang [12] systematically derived tracked vehicle dynamics models which considered the mobility over soft terrains, ride dynamics over rough surfaces and maneuverability. They also studied the ride quality of tracked vehicle over deformable terrains. Dhir and Shankar et al. [13,14] developed a ride dynamic simulation model of a typical tracked vehicle assuming constant vehicle speed and non-deformable terrain surface. The wheel/track-terrain interactions and dynamic track loads were also taken into consideration. Ata et al. [15] conducted a theoretical investigation into the effect of suspension configurations on a tracked vehicle performance over bump terrains. The suspension configurations, especially the numbers and locations of suspension dampers, were found to have a significant effect on vehicle mobility. Thanks to the rapid development of advanced computational tools, such as LMS Motion, Adams ATV and Recurdyn, multi-body dynamics (MBD) simulations have been widely used to rapidly predict the dynamic response of tracked vehicles in distinct virtual environments $[16,17]$.

Unlike large tracked vehicles with ample design space, small and medium-sized tracked vehicles often adopt simple spring-damper suspension or just rigid chassis [18,19]. This paper proposes a novel compact tracked chassis with multi-cylinder hydropneumatic suspension to replace the conventional wheeled chassis of road-rail vehicles. Compared with the chassis driven by rubber wheels, tracked chassis can flexibly cope with the diverse rough terrains along the railway and switch operation modes without terrain restrictions to meet the growing needs of routine inspection and maintenance.

In this paper, a compact tracked chassis with a hydropneumatic suspension system is proposed, modelled, and evaluated. The main contributions are listed as follows:

1. A novel road-rail vehicle equipped with a tracked chassis is designed with the capability of autonomously switching between two operation modes and adapting to the various rugged and uneven terrains along the railway;

2. Lugre friction model is integrated with the hydropneumatic suspension model to improve the model accuracy. The suspension model is experimentally verified by the test rig data for a further establishment of the integral vehicle model;

3. The hydraulic and pneumatic parameters are optimized based on a systematic objective function, which takes into account the heave and pitch motion states for achieving better dynamic performance of the vehicle.

This paper is organized as follows. An overview of the proposed novel road-rail vehicle is presented in Section 2; Section 3 elaborates the design principle and the hydropneumatic suspension modelling. Sections 4 and 5 report the model validation process and the ride performance analysis, respectively; Finally, Section 6 concludes this paper.

\section{Overview of the Road-Rail Vehicle}

The overall compact design of the novel road-rail vehicle can be seen in Figure 1a. As shown in Figure 1a, this proposed vehicle consists of one tracked chassis, one bogie and a transfer module set. Figure $1 b, c$ illustrate that the bogie and the tracked chassis 
are applied as the chassis for rail driving and road driving, respectively. The transfer module is responsible for the switching between two operation modes, which comprises one composite oil cylinder, one rotatable claw and four lift cylinders. The composite oil cylinder connected with claw is mounted on the bogie, which can hold up and rotate the whole vehicle. The lower ends of the lift cylinders are mounted on the tracked chassis, and the upper ends are connected to the bogie so that the lifting cylinders can change the distance between the bogie and the tracked chassis.

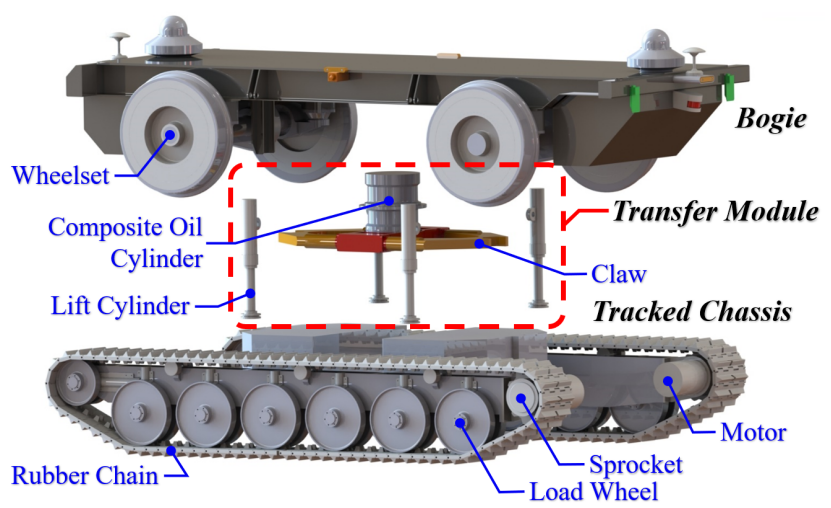

(a)

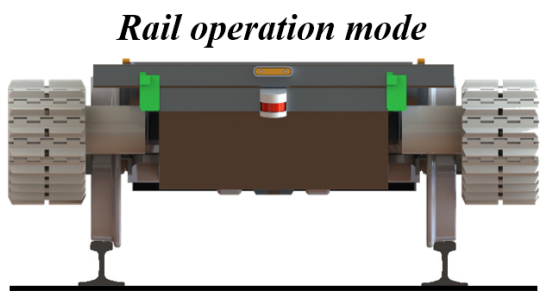

(b)

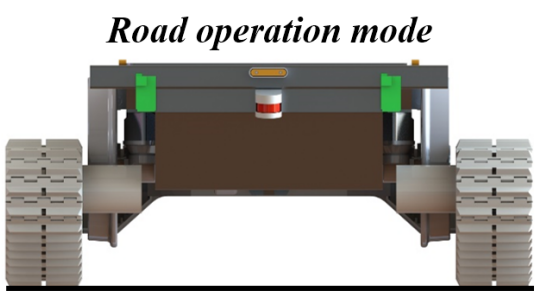

(c)

Figure 1. Proposed road-rail vehicle (a) general view (b) rail operation mode (c) road operation mode.

For the rail operation mode, the wheelsets on the bogie ride on the rails and highpressure oil will be pumped into the lower chambers of the four lift cylinders to lift the tracked chassis (with load wheels and pedrails) to leave the ground.

For the road operation mode, the left and right sprockets are driven by two motors, respectively. High-pressure oil will be pumped into the upper chambers of the four lift cylinders to raise the bogie and ensure the tracked chassis has sufficient ground clearance.

The switching from road operation mode to rail operation mode will be explained in the following. Figure 2a illustrates the body attitude change during the climbing process. The vehicle approaches the rails perpendicularly in road operation mode and moves slowly to reduce the impact. When approaching the railway, the vehicle adjusts its orientation to ensure that both pedrails can touch the rail almost simultaneously. The vehicle continues to move forward after both pedrails contact with the second rail and finally stops when it arrives at the middle of two rails.

Figure $2 b, c$ show the process of operation mode switching. (1) The composite cylinder will lower the claw first. After the claw tightens to the rails, the composite cylinder will raise the whole vehicle. (2) Then four lift cylinders will lift the tracked chassis. The load wheels can be further lifted by the suspension system. (3) After that, the composite cylinder will turn the vehicle by 90 degree to align the vehicle along the rail direction. (4) The claw will be retrieved, and the wheelsets will ride on the rails. The operation mode switching is then completed, as shown in Figure 2d. Next, the vehicle will be driven by the bogie. 


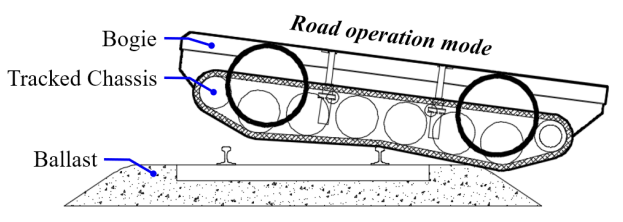

(a)

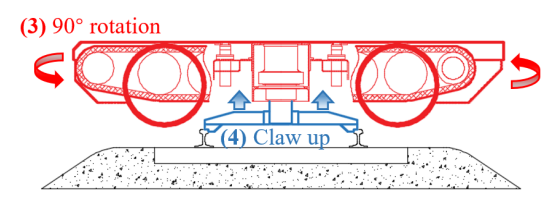

(c)

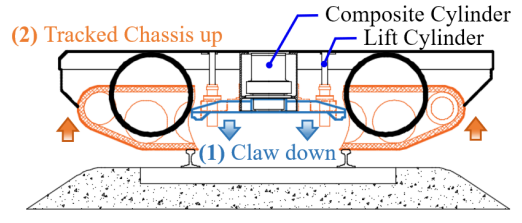

(b)

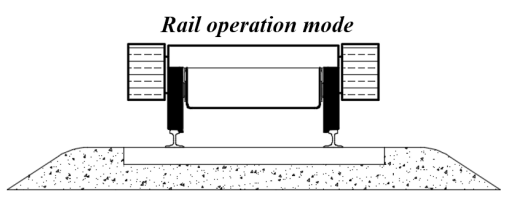

(d)

Figure 2. Operation mode switching process (a) Climbing process (b) Lifting process (c) Rotating process (d) Switching complete.

\section{Suspension Design and Modelling}

This section introduces the design principle and layout of the hydropneumatic suspension system of the tracked chassis. This novel road-rail vehicle has two sets of suspension systems. Since the suspension system of the bogie has been studied in the previous paper [20], the suspension system mentioned in the following all refers to the hydropneumatic suspension system of the tracked chassis.

\subsection{Suspension Design of the Tracked Chassis}

Figure 3 shows the structure of the cylinder-piston assembly and the suspension status in two operation modes. The suspension is mainly composed of an upper pneumatic chamber and a lower hydraulic chamber. The hydropneumatic cylinder-piston assemblies connect the load wheels to the tracked frame. The lower end (piston) is mounted on the U-shaped shaft with a load wheel. The upper end (cylinder) is fixed with the tracked frame. It should be emphasized that the design of the U-shaped shaft increases the total stroke of the suspension compared to the traditional unbent shaft. This design overcomes the height limitation of the tracked chassis and significantly reduces the risk of suspension breakdown.

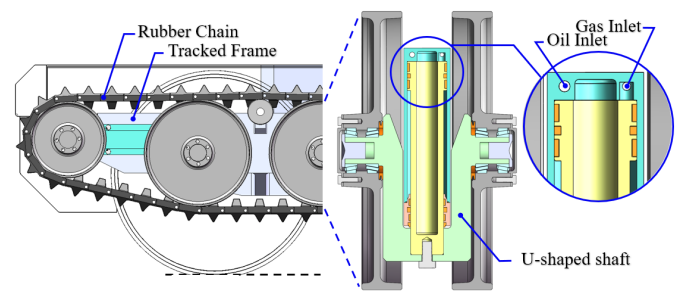

(a)

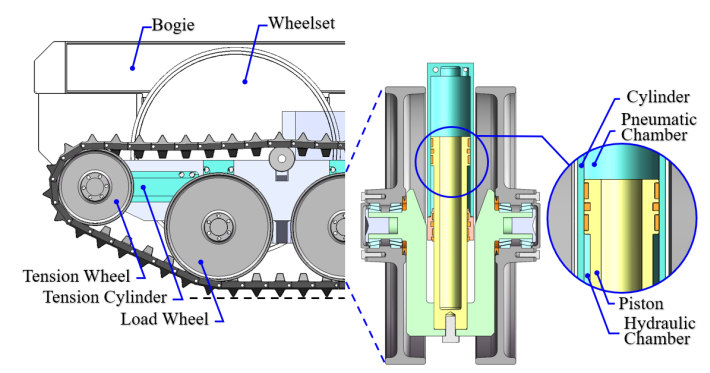

(b)

Figure 3. Tracked chassis suspension status in two operation modes (a) Rail operation mode (b) Road operation mode. 
As shown in Figure 3a, the whole tracked chassis is wholly suspended during the rail operation mode. The oil is injected into the hydraulic chamber as the filling medium and push the piston to the top of the cylinder so that the load wheel does not produce vertical vibration affecting the driving stability of the vehicle.

During the road operation mode as shown in Figure 3b, the pneumatic chamber is filled with nitrogen as an elastic medium, which provides a non-linear suspension stiffness. The hydraulic chamber is filled with oil, which provides a suspension damping. When driving on the ballasts or the terrains beside the rail, the bounce displacement of the load wheel is small, the suspension stiffness is low, and the vehicle has good driving stability. When crossing the barriers such as the rail and the ramp, the load wheel's stroke becomes large, so strong shock absorption capacity and high reliability could be achieved.

The hydropneumatic suspension system of the in-plane model is illustrated in Figure 4. When valve B opens and valves A, C, D close, the hydraulic chamber is directly connected to the oil tank and the suspension system is working in road operation mode. When valves A, C open and valves B, D close, the oil pump supplies oil to the hydraulic chamber and the tension cylinder simultaneously. The gas in the pneumatic chamber is compressed and the load wheels are lifted.

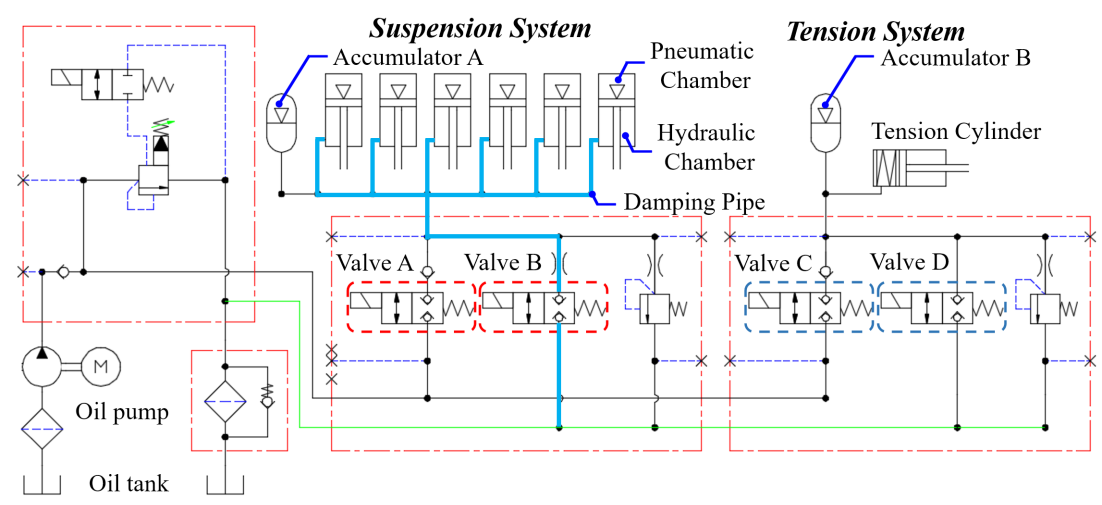

Figure 4. Hydropneumatic suspension system for the half-vehicle.

When the load wheels reach the limit position, valve A closes. The pressure in accumulator A ensures that the load wheels maintain the limit position; The tension cylinder is stretched to ensure that the pedrails are not slack. When the tension force reaches a limit value, valve $C$ closes and accumulator $B$ will hold the tension pressure.

\subsection{Modelling of the Hydropneumatic Suspension}

An entire hydropneumatic suspension system, composed of the pneumatic chamber model, the damping pipe model, and the friction model, is established with non-linear features by AMESim. To study the overall dynamics and discuss the ride performance of the tracked chassis, a single-cylinder model needs to be investigated first.

Many scholars [21-23] have established mathematical models for the pneumatic chamber model and the oil damping model of the hydropneumatic suspension. However, the friction is either neglected or taken as the simple Coulomb friction, which has a significant deviation on the model precision since friction is one of the primary sources of non-linear characteristics in the hydropneumatic suspension system.

\subsubsection{Mathematical Model of the Actuation Cylinder}

Assumptions are Made as Follows in the Proposed Model:

1. All parts of the cylinder have been effectively sealed. No gas dissolved into the oil;

2. The cylinder components are rigid since the working pressure only varies within a range of 40 bar in the pneumatic chamber;

3. The oil is incompressible since the oil is just the damping medium in this system;

4. The mass and the inertia of the oil is negligible. 
The exerted force of suspension in this test rig is related to gas pressure $P_{1}$ in the pneumatic chamber, oil pressure $P_{2}$ in the hydraulic chamber, and seal element friction force $F_{f}$.

$$
F=P_{1} A_{1}-P_{2} A_{2}-F_{f}
$$

where $A_{1}$ and $A_{2}$ are the sectional areas of the pneumatic and hydraulic chambers, respectively.

\subsubsection{Model of the Pneumatic Chamber}

The pneumatic chamber of the cylinder is filled up with pressurized nitrogen and can be regarded as a gas spring. The corresponding gas pressure and gas volume are given by the polytropic process equation:

$$
\begin{aligned}
& P_{1} V_{1}^{n}=P_{0} V_{0}^{n} \\
& V_{1}=V_{0}+\Delta V
\end{aligned}
$$

where $n$ is the polytrophic index, $P_{0}$ and $V_{0}$ are the pre-charge pressure and volume of the pneumatic chamber, $P_{1}$ and $V_{1}$ are the actual initial gas pressure and volume, respectively, $\Delta V$ is the volume change of gas. It needs to be emphasized that $P_{1}$ is determined by the weight of the vehicle, so it is constant in this paper.

If the oil flows quickly with the reciprocating motion of the piston, the polytrophic index $n$ is 1.4 based on the adiabatic thermodynamic process. Inversely, the thermodynamic process is the isothermal process and $n=1$. The polytropic exponent $n$ is set as 1.3 based on the practical application [17].

\subsubsection{Model of Damping Pipe}

The damping force of the suspension system is mainly derived from the pressure loss generated by sudden changes in the flow cross-section. The pressure loss between the hydraulic chamber and the oil tank is composed of the partial pressure loss and the pressure loss along the pipe.

Hydraulic chamber pressure $P_{2}$ can be expressed as:

$$
\begin{gathered}
P_{2}=\rho\left(\frac{Q}{A_{c}}\right)^{2}\left\{\xi_{1}[0.5+0.5 \operatorname{sign}(v)]-\xi_{2}[0.5-0.5 \operatorname{sign}(v)]\right\}+\lambda \frac{l}{d} \frac{\rho V_{c}^{2}}{2} \operatorname{sign}(v) \\
Q=A_{2} v \\
V_{c}=\frac{Q}{A_{c}}=\frac{A_{2} v}{A_{c}}
\end{gathered}
$$

where $\rho$ and $V_{c}$ are the density and flow velocity of the oil, $Q$ is the flow rate, $A_{c}$ is the sectional area of damping pipe, $\xi_{1}$ and $\xi_{2}$ are the pressure distribution coefficient of oil outflow and inflow, $l$ and $d$ are the length and diameter of damping pipe, $v$ is the velocity of the piston relative to the cylinder.

$\lambda$ is the flow restriction coefficient along the pipe:

$$
\lambda=\left\{\begin{array}{lc}
\frac{80}{R e}, & R e<2000 \\
\frac{0.3164}{R e^{0.25}}, & 2000 \leq R e \leq 10^{5}
\end{array}\right.
$$

the Reynolds number is defined as:

$$
R e=\rho \frac{V_{c} d}{\mu}
$$


and the sign function is given as:

$$
\operatorname{sign}(v)=\left\{\begin{array}{cc}
1, & v>0 \\
0, & v=0 \\
-1, & v<0
\end{array}\right.
$$

where $\mu$ is the oil kinematic viscosity. The associated initial values of hydropneumatic variables are listed in Table 1.

Table 1. Initial value of hydropneumatic variables.

\begin{tabular}{cccc}
\hline Hydraulic Variables & Symbol & Unit & Value \\
\hline Pre-charge gas pressure & $P_{0}$ & $\mathrm{~Pa}$ & $7 \times 10^{5}$ \\
Pre-charge gas volume & $V_{0}$ & $\mathrm{~m}^{3}$ & $2.64 \times 10^{-4}$ \\
Length of the damping pipe & $l$ & $\mathrm{~m}$ & 0.5 \\
Diameter of the damping pipe & $d$ & $\mathrm{~m}$ & $7.5 \times 10^{-3}$ \\
Sectional area of the damping pipe & $A_{\mathrm{C}}$ & $\mathrm{m}^{2}$ & $4.4 \times 10^{-5}$ \\
Density of the oil & $\rho$ & $\mathrm{Kg} / \mathrm{m}^{3}$ & $0.87 \times 10^{3}$ \\
Gas polytrophic exponent & $n$ & & 1.3 \\
Kinematic viscosity of the oil & $\mu$ & $\mathrm{Pa} \cdot \mathrm{s}$ & 10 \\
\hline
\end{tabular}

\subsubsection{Lugre Friction Model}

For the hydropneumatic suspension system, the friction force generated by the seal and guiding elements significantly impacts the vibration calculation. Many researchers have studied Coulomb friction characteristic in cylinders or actuators [24], but such as Karnopp and LuGre model $[25,26]$ have been proved to be more effective on many occasions. Yanada and Sekikawa [26] studied the dynamic behaviors of friction using a hydraulic cylinder. They proposed a modification to the LuGre model to improve the simulation accuracy. The LuGre friction model takes the surface contact as the bristles' elastic deformation under the micro-structure as shown in Figure 5. It can provide a comprehensive description of the static and dynamic characteristics of the friction between the piston and the cylinder. Whether the friction model parameters can be effectively and quickly identified from a large amount of experimental data is of great significance for improving the accuracy of the suspension model. This paper chooses the genetic algorithm (GA) to identify the friction model parameters because it has a comprehensive search range and is easy to obtain the optimal global solution.

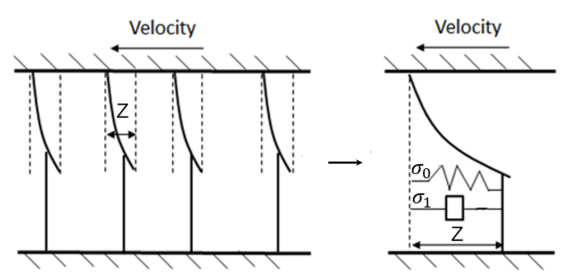

Figure 5. LuGre friction model.

The LuGre model is described by:

$$
\left\{\begin{array}{l}
F_{f}=\sigma_{0} z+\sigma_{1} \frac{d z}{d t}+\sigma_{2} v \\
\frac{d z}{d t}=v-\frac{\sigma_{0} z}{g(v)}|v| \\
g(v)=F_{c}+\left(F_{S}-F_{C}\right) e^{-\left(\frac{v}{v_{s}}\right)^{2}}
\end{array}\right.
$$

where $\sigma_{0}$ is the stiffness for a spring-like behavior for small displacements, $\sigma_{1}$ and $\sigma_{2}$ are the damping coefficient that relates to pre-sliding and viscous friction states, $z$ is the average bristle deflection, $g(v)$ is Stribeck's curve in the function of sliding velocity, $v_{s}$ is the Stribeck velocity, $F_{S}$ and $F_{c}$ are the static friction force and Coulomb force. 
LuGre friction model includes four static parameters and two dynamic parameters. The GA program shown in Figure 6 indicates the whole process of parameter identification. The first step is to identify the static parameters $F_{s}, F_{c}, v_{s}$ and $\sigma_{2}$. In the static case, it is assumed that the bristles' deformation has reached the maximum and that is to say the $\frac{d z}{d t}=0$. In this step, the friction force can be expressed as:

$$
F=\left(F_{c}+\left(F_{s}-F_{c}\right) e^{-\left(\frac{v}{v_{s}}\right)^{2}}\right) \operatorname{sign}(v)+\sigma_{2} v
$$

The dynamic parameters identification is based on the static parameters result. Above four identified static parameters are brought into the Lugre friction model, and then the dynamic parameters $\sigma_{0}$ and $\sigma_{1}$ are also identified by the GA program.

$$
F=\sigma_{0} z+\sigma_{1} v-\frac{\sigma_{0} \sigma_{1} z|v|}{\left(F_{c}+\left(F_{S}-F_{c}\right) e^{-\left(\frac{v}{v_{s}}\right)^{2}}\right)}+\sigma_{2} v
$$

In the Matlab GA program, the maximum generation is 200, the population size is 200, the reproduction ratio is 0.7 , and the mutation probability is 0.03 . The identified values of these parameters are shown in Table 2.

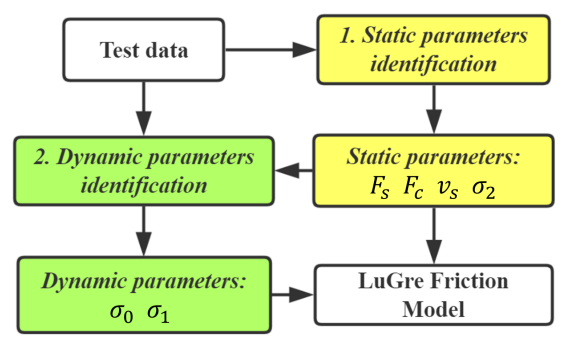

Figure 6. GA program of LuGre model parameters identification.

Table 2. Lugre friction model parameters.

\begin{tabular}{cccc}
\hline Variables & Symbol & Unit & Value \\
\hline Stiffness coefficient & $\sigma_{0}$ & $\mathrm{~N} / \mathrm{m}$ & $5 \times 10^{6}$ \\
Damping of pre-sliding & $\sigma_{1}$ & $\mathrm{~N} /(\mathrm{m} / \mathrm{s})$ & 30 \\
Damping of viscous friction & $\sigma_{2}$ & $\mathrm{~N} /(\mathrm{m} / \mathrm{s})$ & 0.8 \\
Static friction force & $F_{s}$ & $\mathrm{~N}$ & 400 \\
Coulomb force & $F_{C}$ & $\mathrm{~N}$ & 100 \\
Stribeck velocity & $v_{s}$ & $\mathrm{~m} / \mathrm{s}$ & 0.02 \\
\hline
\end{tabular}

\section{Experimental Model Verification}

To validate the suspension model, the bench test of a single-cylinder hydropneumatic suspension shown in Figure 7 is established. The parameters in Tables 1-3 are used.

Table 3. Suspension structure parameters.

\begin{tabular}{cccc}
\hline Structure Parameters & Symbol & Unit & Value \\
\hline Mass of load wheel & $m_{w}$ & $\mathrm{~kg}$ & 16 \\
Mass of piston & $m_{p}$ & $\mathrm{~kg}$ & 4 \\
Hydraulic cylinder inner diameter & $d_{c}$ & $\mathrm{~mm}$ & 60 \\
Piston diameter & $d_{p}$ & $\mathrm{~mm}$ & 50 \\
Stroke of load wheel & $S$ & $\mathrm{~mm}$ & 155 \\
\hline
\end{tabular}




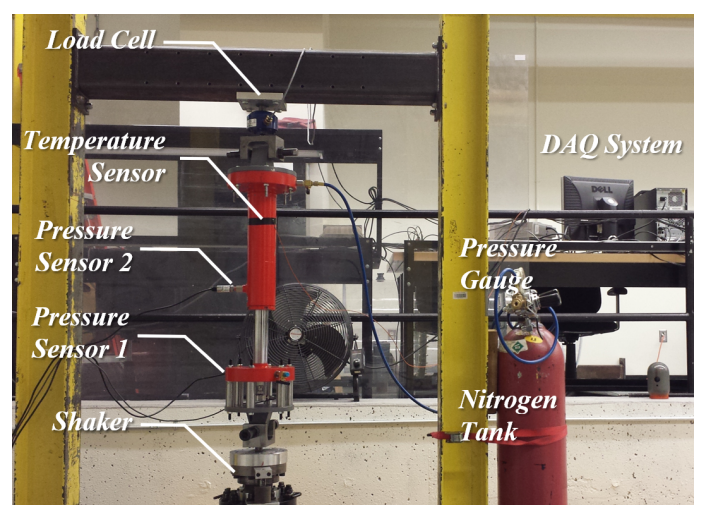

Figure 7. Experimental setup for hydropneumatic suspension.

The top of the cylinder is fixed on a beam. Excitation is applied on the bottom of the piston by a shaker. A load cell is installed between the beam and cylinder. The pressure sensor 1 is installed on the bottom of the piston to measure gas pressure in chamber 1 . The pressure sensor 2 is installed to measure the oil pressure in the hydraulic chamber. The shaker system has displacement and velocity sensors.

This test rig cannot test the friction force directly. Based on the data of load cell, the pressure in two chambers and displacement-velocity, friction force can be calculated accurately. Figure 8 shows the test and model results of the friction force. The experimental data verifies the accuracy of the model of the hydropneumatic suspension.
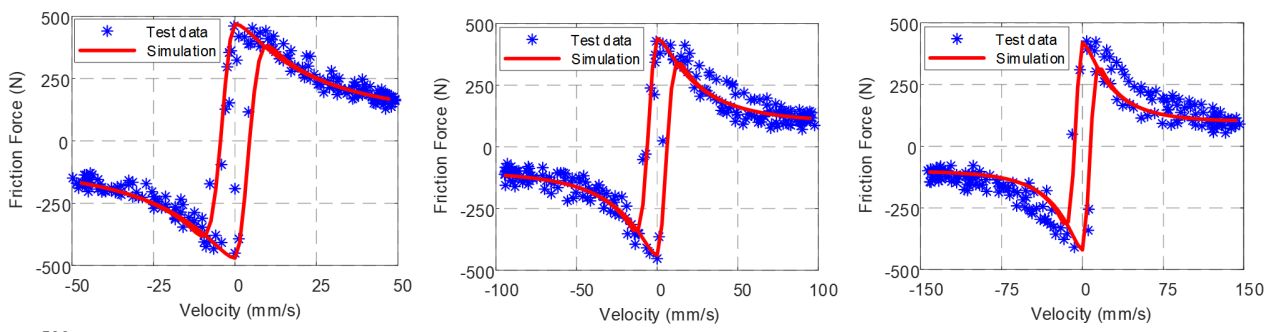

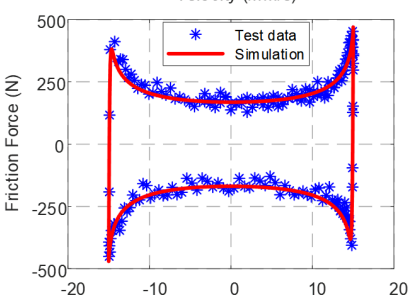

(a)

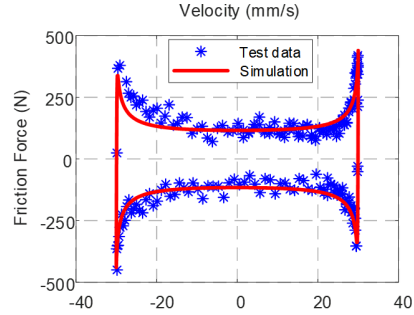

(b)

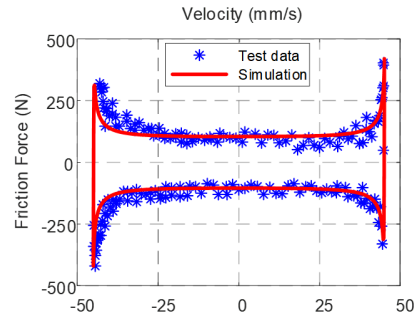

(c)

Figure 8. Friction force versus velocity and displacement under different sinusoidal amplitude (a) $0.5 \mathrm{~Hz} 15 \mathrm{~mm}$ (b) $0.5 \mathrm{~Hz} 30 \mathrm{~mm}$ (c) $0.5 \mathrm{~Hz} 45 \mathrm{~mm}$.

Figure 9 shows the exerted force of the hydropneumatic cylinder at $0.5 \mathrm{~Hz}$ with different amplitude harmonic vibration excitation. When the stroke is small, exerted force versus displacement has an approximate linear characteristic in Figure 9a. However, with the stroke increasing, the exerted force shows power function characteristic. At the ends of each stroke in Figure 9b, the non-linear characteristic of force discontinuity is caused by friction force direction changing. 


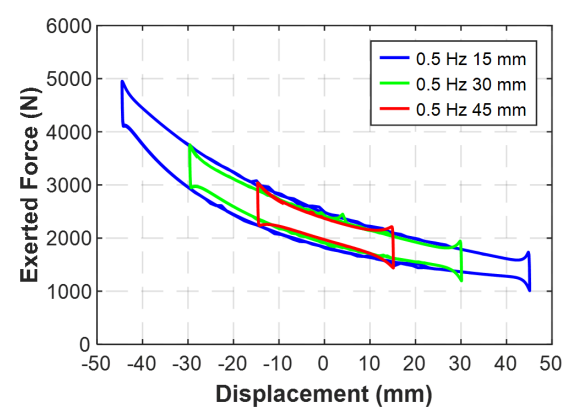

(a)

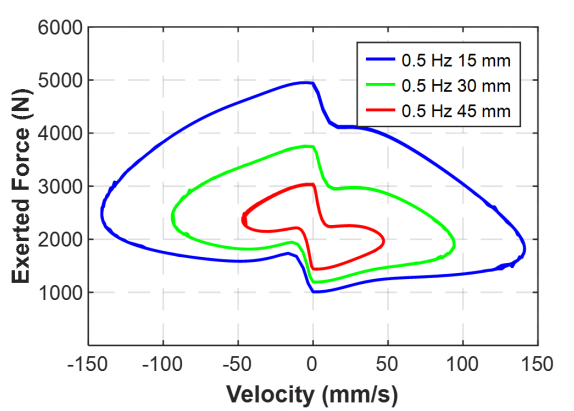

(b)

Figure 9. Exerted force (a) Force-displacement loop (b) Force-velocity loop.

\section{Ride Performance Analysis}

In the railway system, each operating vehicle must strictly follow the schedule. The ride performance is one of the most important indices to keep the working efficiency of the road-rail vehicle to shorten the mode switching time and ensure the safety of onboard equipment.

\subsection{Evaluation Function}

In this work, the suspension performance assessment was carried out by using an evaluation function (EF) that combines both the hull vertical vibration and pitch angle changes. The crest factor (CF) of bounce acceleration (BA), bounce displacement (BD), angular acceleration (AA) and pitch angle (PA) was used to indicate the ride performance improvement. The $\mathrm{CF}$ value is defined as the relation between the peak amplitude (the maximum absolute value) of the signal to the root mean square (r.m.s.) value and offers a quick and accurate prediction of how much impact occurs during periods $[27,28]$. The evaluation function can be expressed as:

$$
\begin{gathered}
E F=\sqrt{\sum_{i=1}^{4} C F_{i}^{2}} \\
C F=\frac{A_{i, p e a k}}{A_{i, r \cdot m s}}, A_{i}=B A, B D, A \cdot \text { and } \cdot P A
\end{gathered}
$$

The vertical and pitch motions are the main criteria in the tracked suspension system design that assess the suspension performance. For better suspension performance, it is required to minimize these criteria [10]. The same weighting factors of $\mathrm{BA}, \mathrm{BD}, \mathrm{AA}$ and PA are chosen due to the constant road roughness and stable speed mentioned below in Section 5.2. The suspension system with a lower EF value is considered the better configuration that enhances the vehicle ride performance.

\subsection{Result Analysis}

As stated in the introduction, many full or half tracked vehicle models have been developed for the systematic evaluation of suspension performance. Furthermore, a twodimensional model of a tracked vehicle is found to be sufficient for detailed analyses of the suspension dynamics and associated shock and vibration environment of the vehicle [14]. Based on the verified single-cylinder model, the half-vehicle hydropneumatic suspension system is established by AMESim, as shown in Figure 10, which is composed of six hydropneumatic suspension units. Moreover, the MBD in-plane model of the vehicle is conducted based on the practical parameters in Table 4 . The data exchange is carried out by the interface module between the two platforms. The aforementioned tension system in Figure 5 is taken as a spring force.

While ensuring the mode switching time in the real scenarios, the pitch and heave vibrations of the tracked vehicle should also be taken into consideration at the same time [29]. The vehicle is designated to traverse two non-deformable specially designed road profile at a constant forward speed of $1 \mathrm{~km} / \mathrm{h}$ to find a trade-off between the work efficiency and 
vehicle stability. The height of the obstacles refers to the size of the China Railway Track System (CRTS) as shown in Figure 11a. The height of the trapezoidal barrier in Figure 11b is the same as the height of the rail track $(176 \mathrm{~mm})$. The height of the step barrier in Figure 11c is the same as the height of the rail board $(200 \mathrm{~mm})$. In the road modelling process, a little inclination to the original vertical road surface can speed up the calculation process and avoid errors reporting without affecting the accuracy of simulation results.

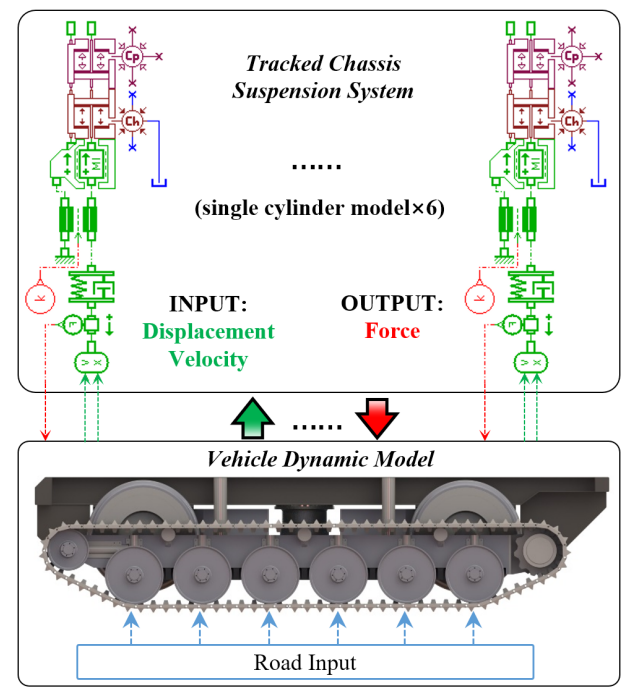

Figure 10. Hydraulic system model of the hydropneumatic suspension.

Table 4. Vehicle parameters.

\begin{tabular}{cccccc}
\hline Structure Parameters & Unit & Value & Structure Parameters & Unit & Value \\
\hline Mass of half-vehicle & $\mathrm{kg}$ & 1250 & Sprocket tooth height & $\mathrm{mm}$ & 28 \\
Length of the chassis & $\mathrm{mm}$ & 3771 & Track width & $\mathrm{mm}$ & 350 \\
Height of the chassis & $\mathrm{mm}$ & 633 & Diameter of tension wheel & $\mathrm{mm}$ & 300 \\
Sprocket pitch & $\mathrm{mm}$ & 90 & Diameter of load wheel & $\mathrm{mm}$ & 420 \\
Sprocket teeth number & & 12 & & & \\
\hline & & & & & \\
& & & & &
\end{tabular}

(a)

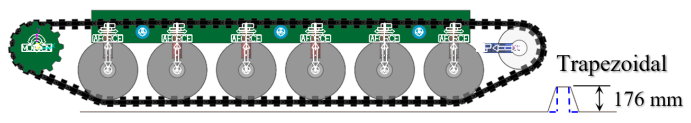

(b)

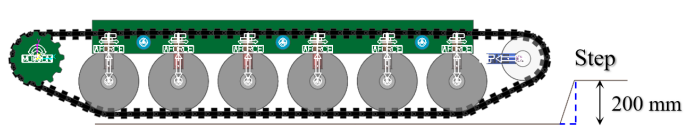

(c)

Figure 11. Road surface establishment (a) CRTSI railway (b) Trapezoidal barrier (c) Step Barrier.

Based on the current compact suspension design space, the size range of the cylinderpiston assembly is already minimal. Therefore, the virtual tests are conducted under the different initial gas volumes $\left(V_{1}\right)$, varied diameters $(d)$ and lengths $(l)$ of the damping pipe. 
The influences of the above parameters on the vehicle ride performance are investigated in the following.

\subsubsection{Effect of the Damping Pipe Diameter}

The damping of hydropneumatic suspension is directly set by the parameters of the pipe installed between the cylinder and the tank. The evaluation function value (EF), bounce acceleration value (BA) and angular acceleration value (AA) for different pipe diameters are obtained and shown in Figure 12, in which the pipe length is $0.5 \mathrm{~m}$ and the pneumatic chamber length is $85 \mathrm{~mm}$.

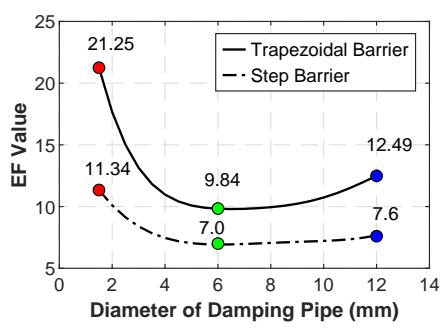

(a)

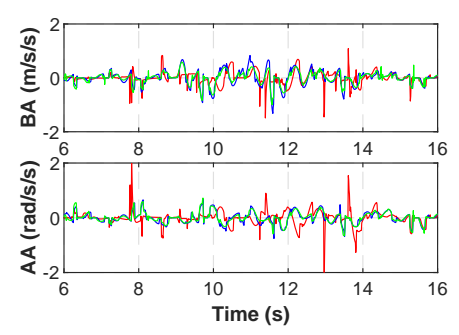

(b)

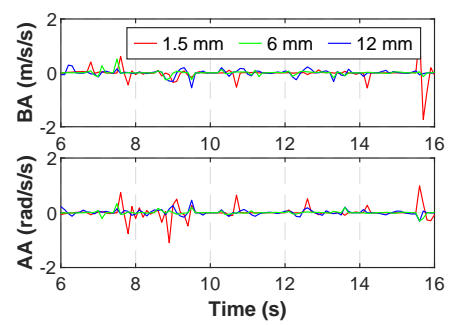

(c)

Figure 12. Performance comparison of different pipe diameters: (a) EF value (b) Acceleration values of trapezoidal barrier traversing (c) Acceleration values of step barrier traversing.

Figure 12a shows that the EF value sharply decreases with the increase of pipe diameter when traversing both the trapezoidal barrier and step barrier. When the pipe diameter is less than $6 \mathrm{~mm}$, the reduction of pipe diameter will lead to a sharp deterioration of the vehicle ride performance in both conditions.

Moreover, the worse road condition, this negative influence is more prominent. When the pipe diameter is $1.5 \mathrm{~mm}$, in the case of the trapezoidal barrier, the EF value once reached 21.25 while the step barrier is only 11.34 at the same traversing speed. This phenomenon can also be seen from the red line in Figure $12 b, c$ that both the angular acceleration value and the vehicle bounce acceleration value have severe fluctuations and shocks. Such large impacts are undoubtedly unacceptable for on-board instruments. The oil flow rate results plotted in Figure 13 shows the oil flow rate of $1.5 \mathrm{~mm}$ diameter is significantly less than the $6 \mathrm{~mm}$ and $12 \mathrm{~mm}$ in both road conditions, which means the pipe cannot function well as a damping unit when the diameter is too small.

When the pipe diameter is larger than $6 \mathrm{~mm}$, further increasing the pipe diameter will slightly increase the EF value, mainly due to the damping force decline caused by the reduction of the flow resistance. Except for the slight difference in peak value, there is not much difference in flow velocity and acceleration between $6 \mathrm{~mm}$ and $12 \mathrm{~mm}$ throughout the barrier traversing process.

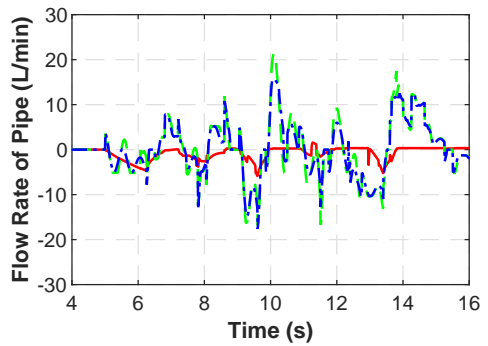

(a)

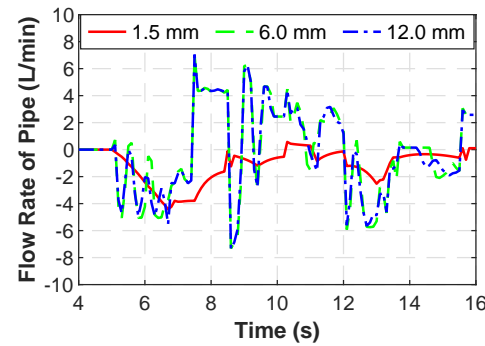

(b)

Figure 13. Flow rate comparison of different pipe diameter: (a) Trapezoidal barrier traversing (b) Step barrier traversing.

Overall, it can be seen from the lower EF value in Figure 12 and lower flow rate in Figure 13 that the body stability when the vehicle traversing a step barrier is much 
smoother than traversing a trapezoidal barrier with similar height and an improperly tuned diameter will definitely cause a severe impact on the vehicle body.

\subsubsection{Effect of the Damping Pipe Length}

The EF, BA and AA value when traversing the trapezoidal barrier and step barrier with different damping pipe length are presented in Figure 14 in which the pipe diameter is $6 \mathrm{~mm}$ and the pneumatic chamber length is $85 \mathrm{~mm}$. The length of the damping pipe will not exceed $2 \mathrm{~m}$ due to the limitations of the interior space. As seen in Figure 14a, the increase of pipe length causes slight growth in EF value and weaken ride performance. Nevertheless, the change in EF value is less than 1 in both road conditions, indicating the length of the damping pipe has a limited influence on vehicle ride performance.

The bounce acceleration and angular acceleration in Figure $14 \mathrm{~b}, \mathrm{c}$ also show that the acceleration of the vehicle body does not change much when the pipe length is $0.1 \mathrm{~m}, 1 \mathrm{~m}$, and $2 \mathrm{~m}$ in both road conditions. Actually, the length of pipe between the cylinders is less than $1 \mathrm{~m}$, so it is not the main factor affecting the ride performance.

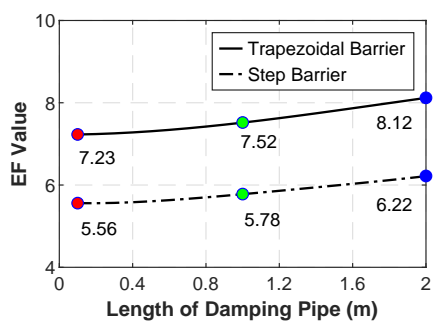

(a)

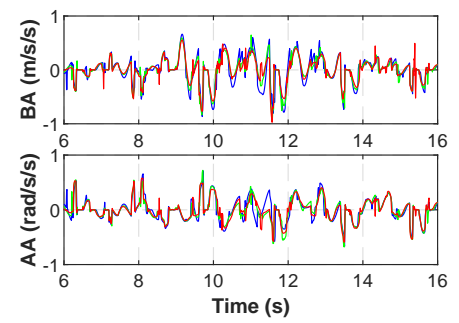

(b)

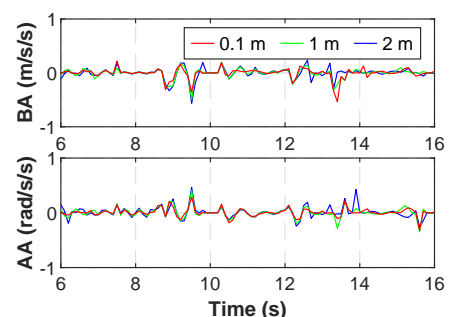

(c)

Figure 14. Performance comparison of different pipe lengths: (a) EF value (b) Acceleration values of trapezoidal barrier traversing (c) Acceleration values of step barrier traversing.

\subsubsection{Effect of the Initial Gas Volume}

Since the sectional area of the piston is constant, different initial gas volumes mean different pneumatic chamber lengths. The EF value is plotted in Figure 15a for different lengths of the pneumatic chamber, in which the diameter and length of the pipe are $6 \mathrm{~mm}$ and $0.5 \mathrm{~m}$, respectively. There exists an optimal length that minimizes the EF value. When the length varies between $50 \mathrm{~mm}$ and $80 \mathrm{~mm}$, the increase of the pneumatic chamber length results in a lower EF value, which indicates a softer suspension system can be obtained by increasing the nitrogen volume. However, on the left side of $50 \mathrm{~mm}$ and the right side of $85 \mathrm{~mm}$, the pneumatic chamber length significantly influences the EF value.

The bounce acceleration of the red curve in Figure 15b indicates that the EF value of Test 1 is mainly caused by the peak acceleration value at $12.21 \mathrm{~s}$. It also can be seen from Figure 16a that when the vehicle traverses the trapezoidal barrier with a $40 \mathrm{~mm}$ pneumatic chamber length, a positive suspension breakdown (the collision between piston and cylinder) occurs at the fourth cylinder. When the third wheel passes through the obstacle, the center of gravity of the vehicle comes to the right side of the barrier, and the balance is instantly altered. During the forward tilt of the body, the fourth load wheel will serve as the only fulcrum to carry the entire weight of the vehicle, thereby triggering a positive impact with an acceleration of $4 \mathrm{~m} / \mathrm{s}^{2}$.

On the contrary, when the vehicle traverses the trapezoidal barrier with a $110 \mathrm{~mm}$ pneumatic chamber length, the negative suspension breakdown occurs as shown in Figure 16b. Due to the large-stroke compression when the front wheels contact with the ground under pitching motion, the pedrail under the fifth and sixth load wheels become slack, thus the collision between the piston and the bottom of the cylinder will happen. Moreover, the clockwise pitch angular acceleration of the vehicle body also aggravates the negative impact of the suspension. 


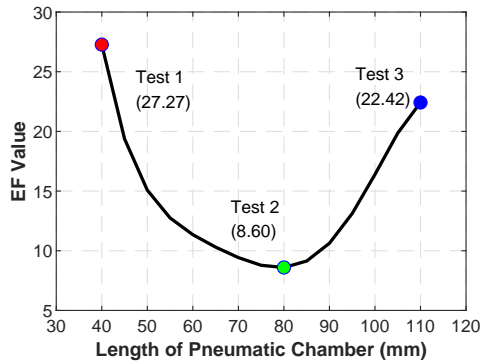

(a)

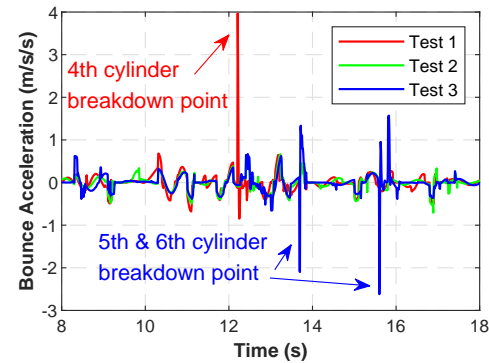

(b)

Figure 15. Performance comparison of different initial gas volumes: (a) EF value (b) Bounce acceleration value.

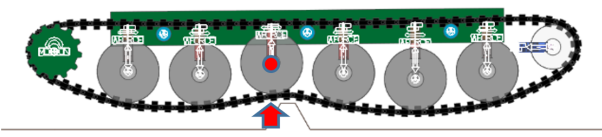

(a)

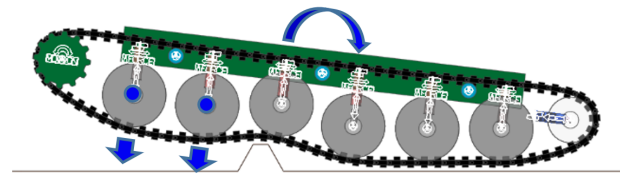

(b)

Figure 16. Suspension breakdown point: (a) Test 1 (b) Test 3.

\section{Conclusions}

This paper has introduced a whole set of hydropneumatic suspension system design for the tracked chassis of a road-rail vehicle that has to adapt to rugged and uneven road surface beside the railway. This hydropneumatic suspension system has been modelled and experimentally verified by the test rig data. One in-plane dynamics model integrated with this hydropneumatic suspension system are established. The influences of the diameter and length of the damping pipe and the initial gas volume on the vehicle ride performance have been analyzed with the systematic objective function.

The test results indicate that the vehicle ride performance is remarkably improved by increasing the damping pipe diameter within a specific range. However, a continued increase in the diameter is not beneficial for further vibration attenuation. The damping pipe length has little effect on the ride performance of this vehicle, but there are still some adverse effects. Hence, the pipe length still needs to be minimized as much as possible. The insufficient gas volume will lead to a severe positive breakdown, especially on the central suspension cylinders, which will bear the entire body weight during the trapezoidal barrier traversing. Increasing the initial gas volume within a specific range can decrease the stiffness of the suspension and is conducive to the improvement of ride performance. However, it also brings the risk of negative breakdown at rear cylinders. That is mainly due to the vast compression of the front cylinders that will loosen the track at the rear. Hence, finding the optimal diameter of the damping pipe and the appropriate gas volume of the hydropneumatic cylinder could effectively enhance the ride performance and ensure vehicle stability and on-board equipment safety.

Based on this research, the next step research will focus on the force control of the tension cylinder, which is meaningful for the improvement of riding performance and reduce the possibility of the suspension breakdown phenomenon.

Author Contributions: Methodology, B.Q.; software, B.Q.; validation, B.Q. and J.Y.; writing-original draft preparation, B.Q.; writing-review and editing, R.Z.; visualization, R.Z.; project administration, J.Y.; funding acquisition, X.L. All authors have read and agreed to the published version of the manuscript.

Funding: This research was funded by CRRC Academy Co., Ltd., grant number No. 2017-529, and the program of China Scholarship Council, grant number No. 201806460055. 
Acknowledgments: This authors would like to thank the CRRC Academy Co., Ltd. and the program of China Scholarship Council for funding this research.

Conflicts of Interest: The authors declare no conflict of interest.

\section{References}

1. Zhang, Z.; Cao, S.; Ruan, C. Statistical linearization analysis of a hydropneumatic suspension system with nonlinearity. IEEE Access 2018, 6, 73760-73773. [CrossRef]

2. Choi, S.B.; Park, D.W.; Suh, M.S. Fuzzy sky-ground hook control of a tracked vehicle featuring semi-active electrorheological suspension units. J. Dyn. Sys. Meas. Control 2002, 124, 150-157. [CrossRef]

3. Rakheja, S.; Afonso, M.; Sankar, S. Dynamic analysis of tracked vehicles with trailing arm suspension and assessment of ride vibrations. Int. J. Veh. Des. 1992, 13, 56-77.

4. Yin, Y.; Rakheja, S.; Boileau, P.E. Multi-performance analyses and design optimisation of hydro-pneumatic suspension system for an articulated frame-steered vehicle. Veh. Syst. Dyn. 2019, 57, 108-133. [CrossRef]

5. Nieto, A.; Morales, A.; Gonzalez, A.; Chicharro, J.; Pintado, P. An analytical model of pneumatic suspensions based on an experimental characterization. J. Sound Vib. 2008, 313, 290-307. [CrossRef]

6. Gobbi, M.; Marinaro, G.; Mastinu, G.; Previati, G. Hydro-Pneumatic Suspension for a $6 \times 6$ All-Terrain Amphibious Vehicle: Design and Testing. In Proceedings of the ASME 2018 International Design Engineering Technical Conferences and Computers and Information in Engineering Conference, American Society of Mechanical Engineers Digital Collection: Quebec, QC, Canada, 26 August 2018.

7. Martini, A.; Bellani, G.; Fragassa, C. Numerical assessment of a new hydro-pneumatic suspension system for motorcycles. Int. J. Autom. Mech. Eng. 2018, 15, 5308-5325. [CrossRef]

8. Cho, J.R.; Lee, H.W.; Yoo, W.S.; Lee, J.K. Study on damping characteristics of hydropneumatic suspension unit of tracked vehicle. KSME Int. J. 2004, 18, 262-271. [CrossRef]

9. Solomon, U.; Padmanabhan, C. Hydro-gas suspension system for a tracked vehicle: Modeling and analysis. J. Terramech. 2011, 48, 125-137. [CrossRef]

10. Wong, J. Dynamics of tracked vehicles. Veh. Syst. Dyn. 1997, 28, 197-219. [CrossRef]

11. Wong, J.Y. Terramechanics and Off-Road Vehicle Engineering: Terrain Behaviour, Off-Road Vehicle Performance and Design; ButterworthHeinemann: Oxford, UK, 2009.

12. McCullough, M.; Haug, E. Dynamics of High Mobility Track Vehicles. J. Mech. Trans. Autom. 1986, 108, 189-196. [CrossRef]

13. Sankar, S.; Dhir, A.; Shankhla, V. Simulation and Field Testing of Tracked Vehicle Suspension Dynamics. J. Dyn. Syst. Meas. Control 1994, 116, 764-773. [CrossRef]

14. Dhir, A.; Sankar, S. Ride dynamics of high-speed tracked vehicles: simulation with field validation. Veh. Syst. Dyn. 1994, 23, 379-409. [CrossRef]

15. Ata, W.; Oyadiji, S. An investigation into the effect of suspension configurations on the performance of tracked vehicles traversing bump terrains. Veh. Syst. Dyn. 2014, 52, 969-991. [CrossRef]

16. Rubinstein, D.; Hitron, R. A detailed multi-body model for dynamic simulation of off-road tracked vehicles. J. Terramech. 2004, 41, 163-173. [CrossRef]

17. Han, S.; Chao, Z.; Liu, X. Research on the effects of hydropneumatic parameters on tracked vehicle ride safety based on cosimulation. Shock Vib. 2017, 2017, 1256536. [CrossRef]

18. Sebastian, B.; Ben-Tzvi, P. Support vector machine based real-time terrain estimation for tracked robots. Mechatronics 2019, 62, 102260. [CrossRef]

19. Zeng, R.; Kang, Y.; Yang, J.; Wang, Z.; Li, G.; Cao, D. Learning-based Terrain Identification with Proprioceptive Sensors for Mobile Robots. IEEE Trans. Ind. Electron. 2020. [CrossRef]

20. Sun, N.; Zhang, W.; Yang, J. Simulation Analysis of a Dual-Purpose Intelligent Mobile Platform for Highway and Railway. SAE Tech. Pap. 2019, 1, 1499.

21. Cao, D.; Rakheja, S.; Su, C.Y. Roll-and pitch-plane coupled hydro-pneumatic suspension: Part 1: Feasibility analysis and suspension properties. Veh. Syst. Dyn. 2010, 48, 361-386. [CrossRef]

22. Kang, Y.; Zhang, W.; Rakheja, S. Relative kinematic and handling performance analyses of independent axle suspensions for a heavy-duty mining truck. Int. J. Heavy Veh. Syst. 2015, 22, 114-136. [CrossRef]

23. Chen, Y.; Hou, Y.; Peterson, A.; Ahmadian, M. Failure mode and effects analysis of dual levelling valve airspring suspensions on truck dynamics. Veh. Syst. Dyn. 2019, 57, 617-635. [CrossRef]

24. Palomares, E.; Nieto, A.; Morales, A.; Chicharro, J.; Pintado, P. Dynamic behaviour of pneumatic linear actuators. Mechatronics 2017, 45, 37-48. [CrossRef]

25. Guo, P.; Lang, Z.; Peng, Z. Analysis and design of the force and displacement transmissibility of nonlinear viscous damper based vibration isolation systems. Nonlinear Dyn. 2012, 67, 2671-2687. [CrossRef]

26. Yanada, H.; Sekikawa, Y. Modeling of dynamic behaviors of friction. Mechatronics 2008, 18, 330-339. [CrossRef]

27. Boileau, P.E.; Turcot, D.; Scory, H. Evaluation of whole-body vibration exposure using a fourth power method and comparison with ISO 2631. J. Sound Vib. 1989, 129, 143-154. [CrossRef] 
28. Pan, H.; Sun, W. Nonlinear output feedback finite-time control for vehicle active suspension systems. IEEE Trans. Ind. Inform. 2018, 15, 2073-2082. [CrossRef]

29. Ning, D.; Du, H.; Zhang, N.; Sun, S.; Li, W. Controllable electrically interconnected suspension system for improving vehicle vibration performance. IEEE/ASME Trans. Mechatron. 2020, 25, 859-871. [CrossRef] 